\title{
RAPS, radio propagation simulator for CBTC system
}

\author{
J. Liang ${ }^{1}$, J. M. Mera ${ }^{3}$, C. Briso ${ }^{3}$, I. Gómez-Rey ${ }^{3}$, A. Garcerán ${ }^{3}$, \\ J. Maroto ${ }^{3}$, K. Katsuta ${ }^{2}$, T. Inoue ${ }^{1} \&$ T. Tsutsumi ${ }^{1}$ \\ ${ }^{1}$ Hitachi, Ltd. Rail Systems Company, \\ Mito Rail Systems Product Division, Japan \\ ${ }^{2}$ Hitachi, Ltd., Hitachi Research Laboratory, Japan \\ ${ }^{3}$ Railway Technology Research Centre, \\ Universidad Politécnica de Madrid, Spain
}

\begin{abstract}
CBTC is Communication-Based Train Control system which is a cost-effective solution for signalling and traffic control of metro and monorail. For the CBTC system that can enhance transportation capacity by moving block control method with less trackside equipment, the radio transmission system is one of the most significant subsystems. Especially, the design and location of all radio-electrical elements is the key factor for the system reliability and availability. Therefore RAPS (Radio Propagation Simulator for CBTC Systems) has been developed together with CITEF (Railway Technology Research Centre of the Universidad Politécnica de Madrid), for the purpose of calculating the radio-electrical signal coverage and power along the track, and arranging a proper layout plan of trackside base stations and associated antennas for each project, in consideration of various aspects, such as tunnel geometry, track configuration, environment affecting obstacles.

The simulator considers all the characteristics of the trackside, like tunnel detailed geometry, open areas cartography and computes propagation according to the specific radio parameters. RAPS is provided with a complete set of design tools, radio-electrical algorithms such as modal propagation for tunnels and Okumura-Hata and Ray-tracing for open areas providing a high accuracy in the prediction of the signal strength along the track. Also user-friendly graphical
\end{abstract}


interface, which is able to make it easy for user to simulate and compare radio wave propagation under many different conditions. This paper shows technical features, graphical user interface and case study of this simulator.

Keywords: CBTC, radio propagation, tunnels, ray tracing.

\section{Introduction}

CBTC is a railway signalling system that makes use of the radio communications between the train and track equipment for the traffic management and infrastructure control [1]. The CBTC systems integrate a digital networked radio system by means of antennas for the bi-directional communication between the trackside equipments and the trains. The radio system is using open ISM radio bands $(2.4 \mathrm{GHz}$ or $5.8 \mathrm{GHz})$, and the transmitter power is limited by the nation's radio law, for example EU limits output power to $100 \mathrm{~mW}$, and Japan is $10 \mathrm{~mW}$. The ISM band is also used in these systems (same as wireless LAN, WiFi), so electromagnetic interference, weak signal strength or saturation of the communications medium are easy to be caused and lead to disrupt the communications. To ensure a stable and reliable radio communication, one of the key questions is the design and location of the trackside base stations and antennas. The precise location of antennas can have a big influence on the quality of the radio bi-directional communication, and the number of antennas or base stations can affected directly the cost of CBTC system. So we need a radio propagation simulator to estimate the optimal numbers and location of base stations by simulating the behaviour of radio waves between transceivers and receivers.

The radio propagation simulator for CBTC (hereafter referred to as RAPS) developed by Hitachi and CITEF enables signal power calculation and helps in the designing and optimization of the elements regarding to the communication and signalling aspects. RAPS is used to provide a platform for making approximate calculations of the signal power and coverage of radio signals within railway environments preconfigured for railway communication systems.

In this paper we present technical features, graphical user interface and case study of RAPS.

\section{RAPS}

\subsection{The outline and configuration}

RAPS is a software application to obtain the power and signal coverage along the central axis of a railway track environment. With a virtual representation of the main aspects of railway environments both in tunnel and open air scenarios, RAPS simulates a particular layout of predefined base stations and associated antennas and calculates an estimation of the radio-electrical signal coverage and power along the track. 
These calculations are stored in a central Data Base and enable the user to create a complete set of data that can be reviewed and analyzed. With the easyto-use RAPS operation concept the user can change all the relevant elements of the scenario (track configuration, environment affecting obstacles, base station and antennas characteristics and locations) and make fast calculations in order to get a clear concept about how many, which and where should be the radioelectrical elements to be displayed on the scenario to grant the specified quality signal levels.

To achieve such complex task, RAPS has been provided with a complete set of designing tools and solving radio-electrical algorithms such as Modal Analysis for tunnels and, Okumura-Hata and Ray-tracing for open areas.

RAPS can manage two types of environments: Pure tunnel scenarios and Open air scenarios.

\subsection{Calculation algorithms module}

\subsubsection{Pure tunnel scenarios}

A tunnel behaves like a hollow wave guide full of a dielectric (air). In this context the modal theory will be used to characterise the electromagnetic propagation within its interior [2]. Accurate modal analysis has been used [3], which enables the propagation to be evaluated with different polarisations, vertical, horizontal and circular, different antenna position for train and base station, and considering correction parameters like the characteristics of the walls of the tunnel, geometry, etc.

Modal analysis is not adequate to analyze more complex environments in tunnels, such as curves and bifurcations. Therefore, another approach based on ray tracing is used on these points [4]. Approximations made by this method enable faster computation times than traditional ones, and this approach does not require an accurate model of the geometry of the environment in tunnels, but it allows finding an approximate result using some basic parameters which characterize curves or bifurcations, such as radius of curvature, building materials, or length of curve.

We considered four types of corrections:

Correction of attenuation due to roughness of the walls

-Type of track: concrete or ballast

$\checkmark$ Effects of catenaries (rigid and flexible)

$\checkmark$ Position of transmitting and receiving antenna

These corrections improve the accuracy of the model.

\subsubsection{Open air scenarios}

Geometric Optics offers a suitable approach with respect to electromagnetic propagation if and when the wavelength is significantly less than the physical dimensions of the elements making up the study scenario. In the model the electromagnetic waves appear as rays which propagate themselves in a straight 
line and have an effect upon, reflect and refract on the surfaces of the elements with which they interact. The accuracy of the results obtained using ray-tracing is determined by the precision with which the geometrical model is built and the number of rays launched or the angular separation between two adjacent rays, as well as frequency [4].

This approach is appropriate for complex situations, such as curved tunnel sections and open areas. In the case of open areas this approach will enable an open space propagation-based approach to be complemented and corrected [5, 6]. This complementing process will be a determinant factor in the event of there being no line of sight.

-Direct ray model

The direct ray model permits to compute the propagation when there is line of sight vision between the transmitter and the receiver.

-Model of reflected rays

The problem of computing several reflections on different objects of the surrounding environment it is complex and there are several approaches. The scenery used to model propagation is based in the use of a high resolution digital cartography where objects are modelled with triangle that defines the different surfaces of the environment.

-Diffracted ray model

The diffraction pattern used is the edge diffraction and leads to an attenuation coefficient depending on the depth of direct ray lock.

The diffraction pattern is not only applicable to the direct beam but also to the reflected rays. Specifically, the calculation of diffraction by obstacles has also been extended to the path of the beam reaches the receiver from the point of reflection, but not the path that starts at the transmitter and ends at the point of reflection.

\subsection{GUI (graphical user interface)}

RAPS is designed on the basis of an easy-to-use working environment for the user. The complete configuration and designing of a railway environment, both underground and open air, involves the use and introduction of many diverse information and parameters. Besides, many pieces of information are available from many different sources with different resolutions. The track geometrical configuration, the constructive definitions of it, the cartography provided for open air environments are all data that must be adapted to be used together.

Once an environment is created, it will be used to make some repeated calculations with different radio-electrical elements configuration in order to study the optimal layout. RAPS' GUI has been designed to achieve this working procedure with ease. 


\subsubsection{Pure tunnel scenarios}

These scenarios consist of tunnel sections with no open-air segments and represent underground railways. RAPS provide tools to design accurate layouts of the track based on six working scopes:

-Horizontal track definition: the geometrical definition of the track in terms of straights, curves and clothoids.

-Vertical track definition: the slope definition for all segments along the track.

-Tunnel sections definition: the definition of tunnel types, dimensions, material and roughness.

-Basement type definition: the definition of basement construction like ballast or slab.

- Overhead contact wire definition: the definition of the catenary type (rigid or flexible).

- Radio-electrical elements definition:

This involves the definition of base station's characteristics and locations along the cross-section of the tunnels and the configuration of associated antennas. This forms the radio-electrical layout for the scenario.

Fig. 1 and Fig. 2 illustrate some samples of tunnel scenarios' GUI.

On the right hand side of the screen (Fig. 1) a form has been set showing graphical information about the scenario.

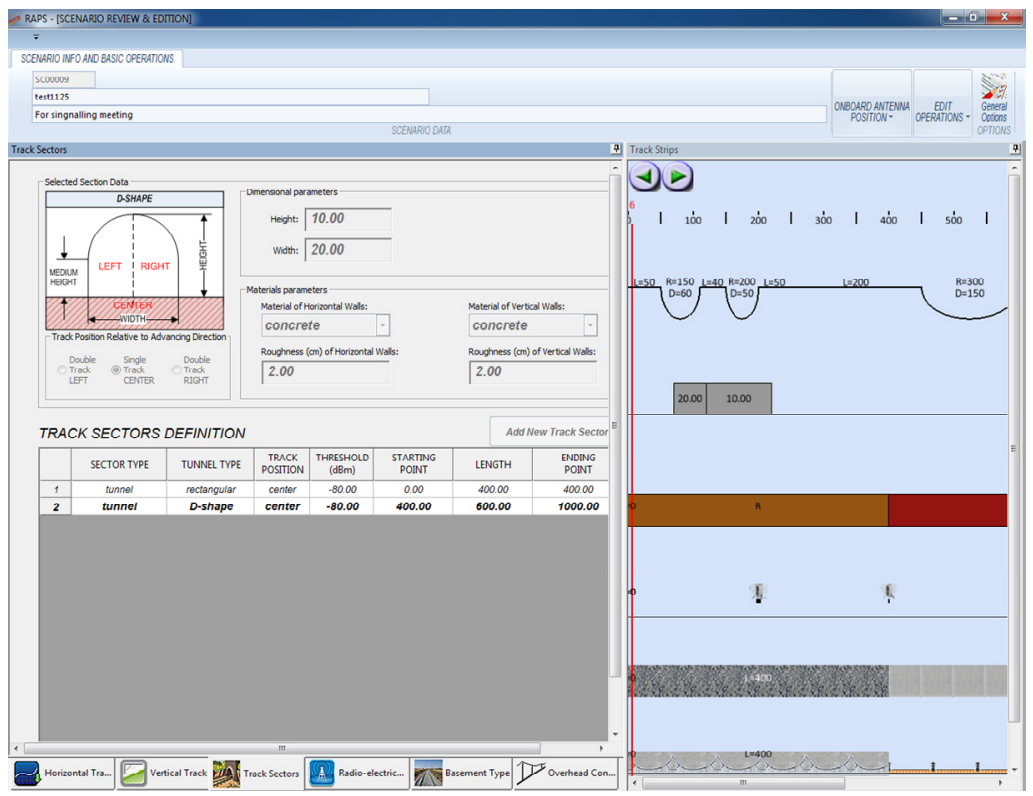

Figure 1: The GUI of tunnel section definition. 


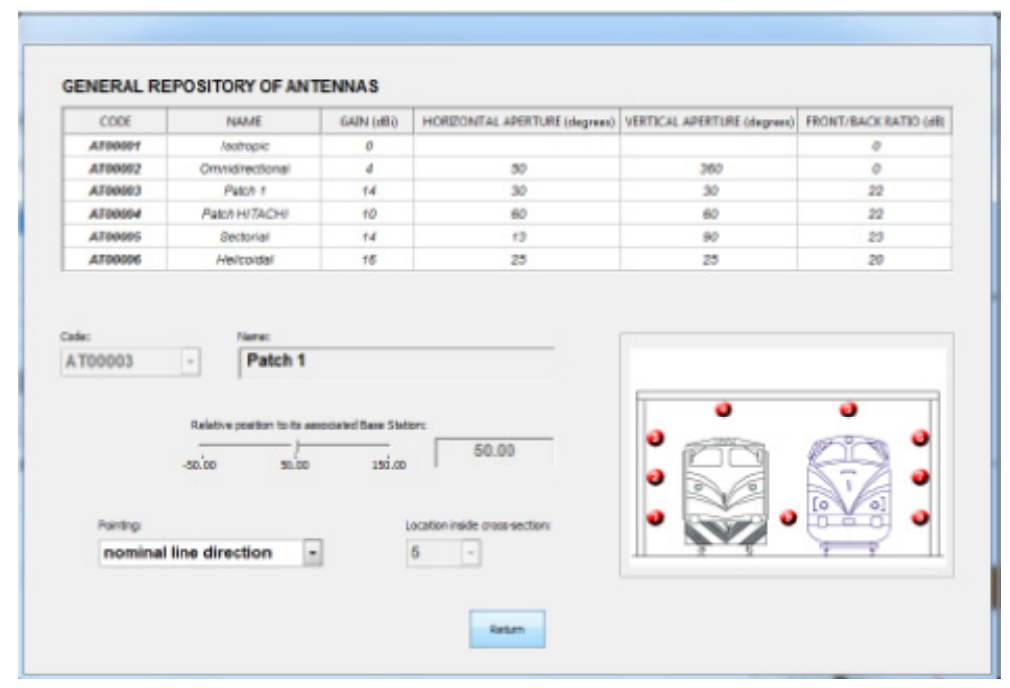

Figure 2: The GUI of antenna configuration and location.

\subsubsection{Open air scenarios}

These are scenarios of open air environments, with or without tunnels. RAPS approaching to the management of such environments include:

-Loading of desired cartography:

The graphical representation of the landscape where the track will be designed is achieved by the loading of DEM (Digital Elevation Model) files and the mapping of aerial photographs inside the application for appropriate visual reference.

- Graphical track definition:

Using a reduced toolset the user can insert, move, edit and delete track points to visually create the desired track on the surface map. Special sections like tunnels can also be configured along the track.

- Graphical environment objects definition:

To configure and locate environment elements over the map which may produce a significant effect on the radio-electrical signal. These should be tree blocks, solid buildings or structures and blocks of randomly distributed buildings.

- Radio-electrical elements definition:

The user can set base stations on any location of the map. A set of associated antennas can be assigned to these base stations. If selected antennas are pointing ones, the user can make a graphical pointing on the map.

Fig. 3 and Fig. 4 illustrate some samples of open air scenarios' GUI. 


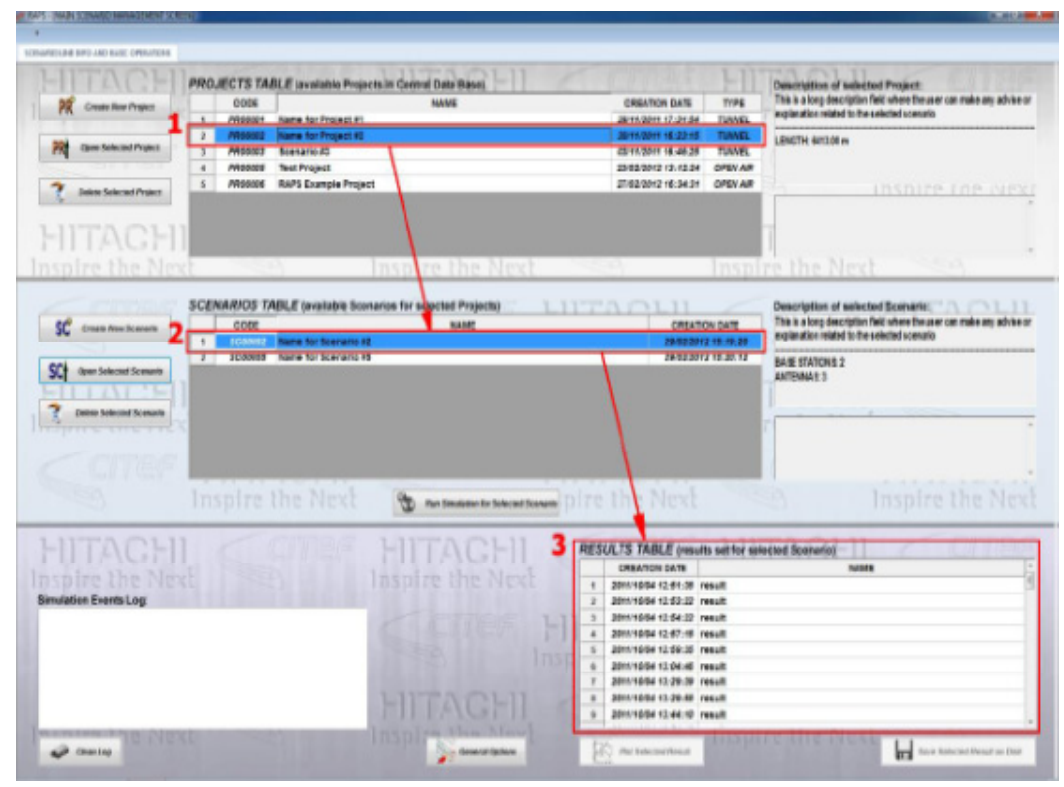

Figure 3: RAPS' GUI main screen.

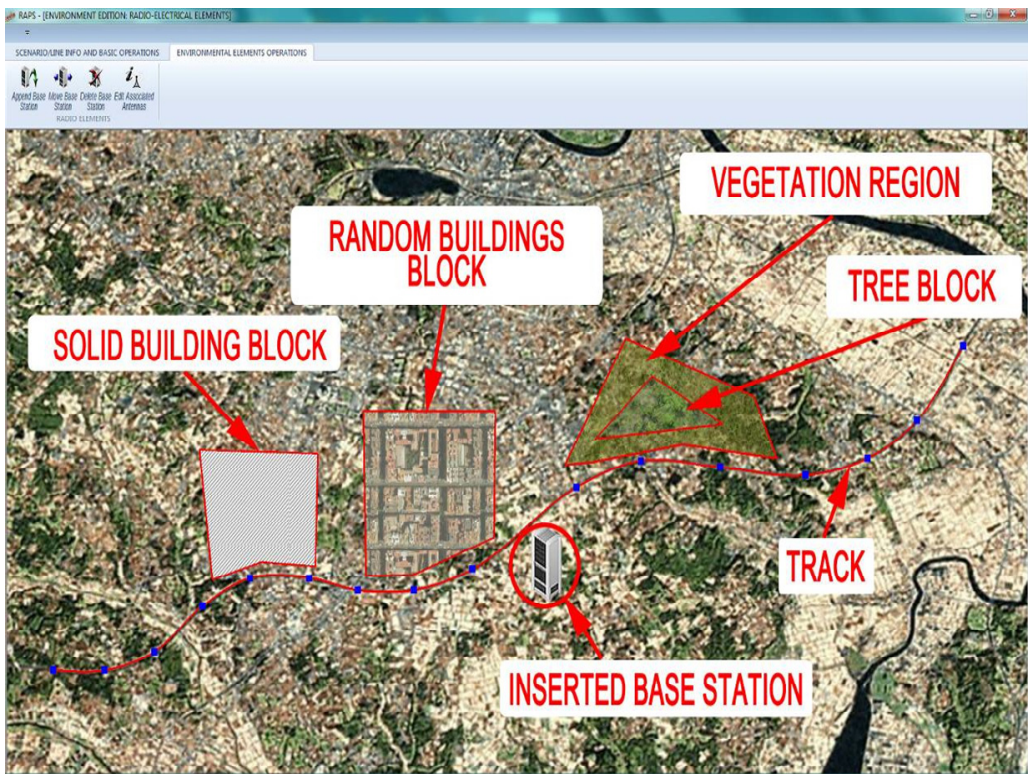

Figure 4: The GUI of track and environment definition. 


\section{Validation}

The estimation and validation of the accuracy of RAPS is one of the most important and complex points. The accuracy has to be evaluated in two regions: tunnels and open areas, and the comparison is made by taking measurements in well defined conditions and environments and comparing the results with data obtained from the simulator. The measurements are statistically compared every 10-30 meter in tunnels and every 100-300 meters in open areas providing a detailed indicator of the accuracy of the simulator.

\subsection{Tunnels validation}

The validation of propagation in tunnels has been made using available measurements made in Madrid subway. Measurements have been taken in different tunnels and conditions using proprietary continuous wave transmitter installed in the walls of the tunnels. The transmitter characteristics are:

The results of one of the measurements it is shown on Figure 5. The figure shows the comparison of measurements and simulations for a smooth curved (radius $=425 \mathrm{~m}$, length $=600 \mathrm{~m}$ ) modern tunnel.

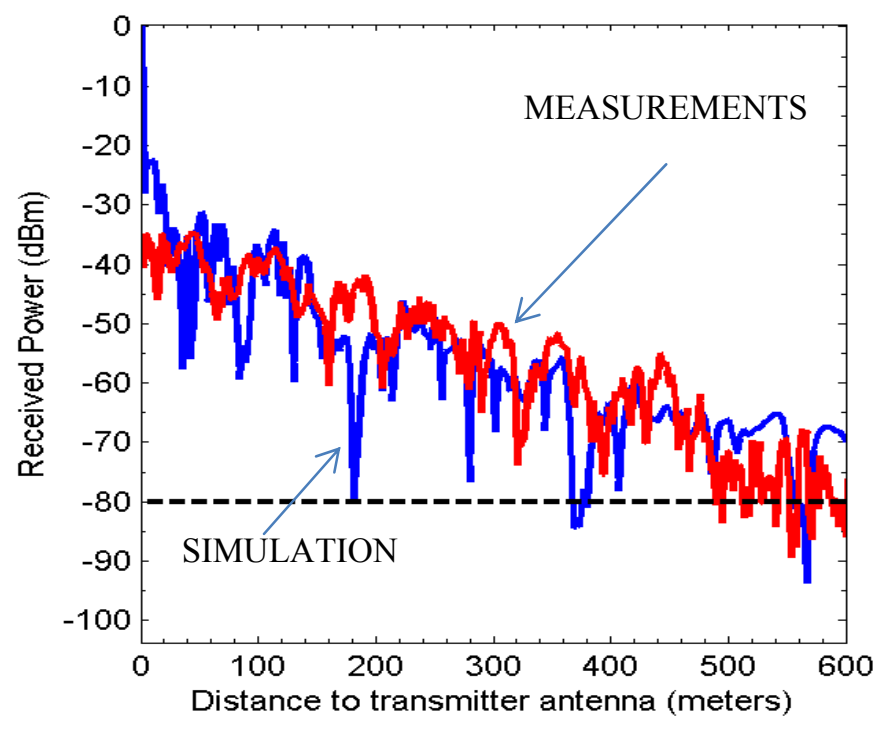

Figure 5: Measurements and simulations in tunnels.

The measurements were compared with simulations of the tunnel [7]. The signal power along track was obtained and statistically compared with measurements made every $1 \mathrm{~m}$. The results provide a high degree of accuracy, for 
both standard deviation and mean value of the signal. Also the signal power above a reference level was obtained with high accuracy. This parameter is commonly used to define the maximum range of one base station, and therefore it is very interesting for the planning of a radio network.

Table 1: $\quad$ Statistical comparison of measurements and simulations in tunnels.

\begin{tabular}{|l|c|c|c|}
\hline & Measurements & Simulation & Difference (\%) \\
\hline Mean value & $-57.5 \mathrm{~dB}$ & $-57.4 \mathrm{~dB}$ & $0.2 \%$ \\
\hline Standard deviation & $14.1 \mathrm{~dB}$ & $14.0 \mathrm{~dB}$ & $1.2 \%$ \\
\hline $\begin{array}{l}\text { Signal > -50dBm } \\
\text { (reference) }\end{array}$ & $92.9 \%$ & $96.0 \%$ & $3.1 \%$ \\
\hline
\end{tabular}

\subsection{Open areas validation}

The second part of the validation is the open area simulators. For this case we made measurements in open areas with an equipped van. The measurements were made in the university campus (Fig. 6).

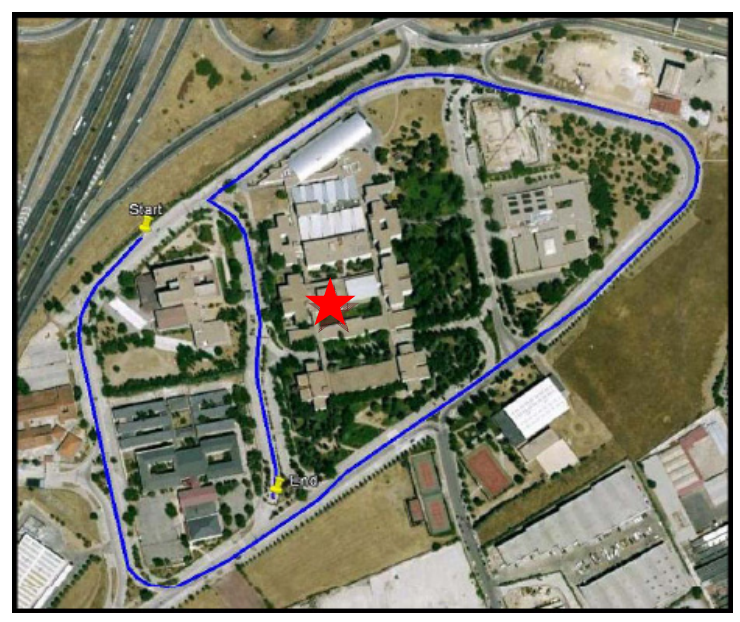

Figure 6: Measurements in the university campus.

With the data of the measurements we reproduce the environment including the main buildings, trees and other small objects. We have compared the measured data with our simulation to estimate the quality of the simulation [8] (Fig. 7). 


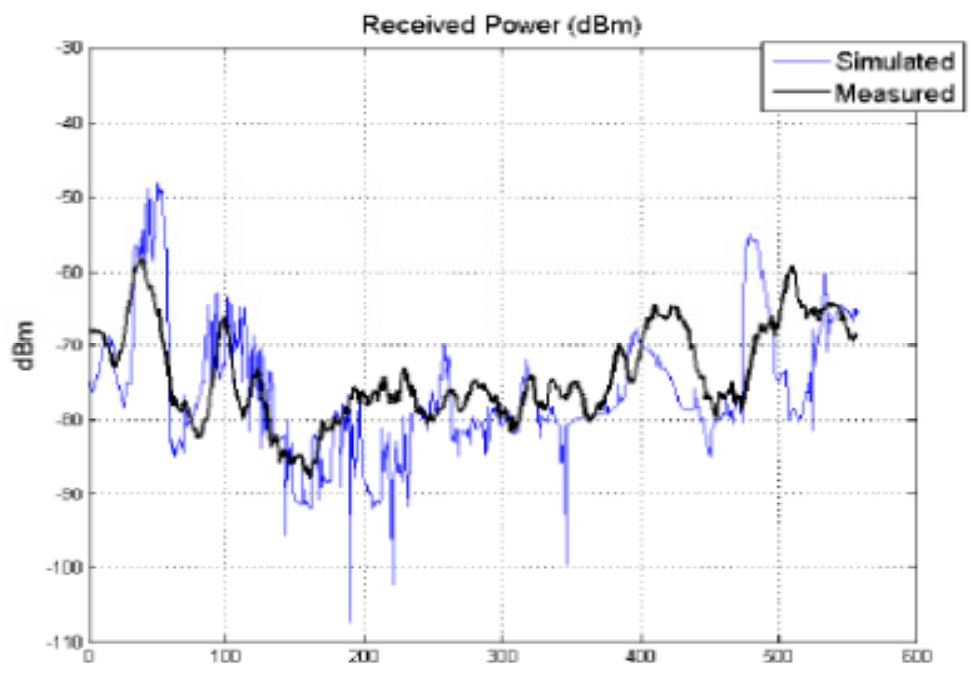

Figure 7: The results of measurements and simulations. Power against the distance.

Average signal power measured $=-72 \mathrm{dBm}$.

Average signal power simulated $=-76 \mathrm{dBm}$.

Mean error $=6 \%$.

\section{Conclusions}

The use of propagation simulators is necessary for the design of complex radio networks for subway environment with tunnels and in dense urban environments. Nevertheless, the railway environment and requirements of the radio network are very different from other commercial applications, so that it is necessary to use specific simulators for the design of this network.

The developed RAPS software uses two fast and accurate algorithms to simulate the propagation in tunnels and urban environment computing the signal strength along track of a complete radio network. The fast and accurate algorithms can simulate radio wave propagation in track environments, including important effects like multi-path propagation due to reflection and diffraction in buildings and objects.

The simulator has been validated using available measurements made in subway tunnels and open areas. The measurements and simulations have shown a high degree of agreement.

As future work, the simulator will be optimized with more measurements taken in tunnels and open areas to improve the accuracy of RAPS and to accumulate know how on using RAPS in real conditions 


\section{References}

[1] IEEE standard for Communications-Based Train Control (CBTC) performance and functional requirements, 30. Dec. 1999.

[2] D. Didascalou, J. Maer, and W. Wiesbeck, "Subway tunnel guided electromagnetic wave propagation at mobile communications frequencies," IEEE Trans. Antennas Propag., Vol. 49, pp. 1590-1596, Nov. 2001.

[3] N. Monk and H. S. Winbigler, "Communication with moving trains in tunnels," IRE Trans. Veh. Commun., vol. PGVC-7, no. 1, pp. 21-28, Dec. 1956.

[4] Zhang, Z.; Yun, Z.; Iskander, M.F. "Ray tracing method for propagation models in wireless communication systems". Electronics Letters .Vol. 36, Issue: 5. 2000, Page(s): 464-465.

[5] Erricolo, D.; Uslenghi, L.E. "Two-dimensional simulator for propagation in urban environments" Vehicular Technology, IEEE Transactions. Vol. 50, Issue: 4. 2001, pp. 1158-1168.

[6] Corre, Y.; Lostanlen, Y. "Three-Dimensional Urban EM Wave Propagation Model for Radio Network Planning and Optimization Over Large Areas". Vehicular Technology, IEEE Transactions. Vol: 58, Issue: 7 .2009, Page(s): 3112-3123.

[7] C. Briso-Rodriguez, J.M. Cruz and J.I. Alonso, "Measurements and Modeling of Distributed Antenna Systems in Railway Tunnels," Veh. Technol., IEEE Trans, vol. 56, Issue 5, Part 2, pp. 2870-2879, Sept. 2007.

[8] M. V. S. N. Prasad and R. Singh, "Terrestrial mobile communications train measurements in Western India," IEEE Trans. Veh. Technol., vol. 52, no. 3, pp. 671-682, May. 2003. 